\title{
Results of cataract surgery in young children in east Africa
}

\author{
David Yorston, Mark Wood, Allen Foster
}

\begin{abstract}
Background-Cataract is the leading cause of blindness in children in east Africa. The results of surgery are poor, partly because of inadequate correction of aphakia.
\end{abstract}

Methods-A retrospective survey of 118 eyes in 71 children with bilateral cataract. All eyes had implantation of an IOL at the time of cataract surgery. The average age at surgery was 3.5 years. 28 patients $(39 \%)$ were less than 2 years old at the time of surgery on their first eye.

Results-Preoperatively, $75.4 \%$ of eyes and $76.1 \%$ of patients were blind. A follow up of at least 3 months was available in 91 $(77.1 \%)$ eyes. In these eyes, $44 \%$ had a latest corrected vision of $6 / 18$ or better and $91.2 \%$ had a latest corrected vision of $\mathbf{6 / 6 0}$ or better. Eyes with zonular cataract, and eyes operated after the age of 2 years were more likely to obtain a vision of $6 / 18$ or better. $3.3 \%$ of eyes and $1.8 \%$ of patients had an acuity of less than $3 / 60$. Nystagmus was present in $42.3 \%$ of patients before surgery. In those patients followed up for a minimum of 6 months, $10.2 \%$ still had nystagmus. The most frequent complication was severe fibrinous uveitis, which occurred in $36(30.5 \%)$ eyes. $62(52.5 \%)$ eyes had a posterior capsulotomy at the time of cataract extraction. Of the remaining 56 eyes, $20(35.7 \%)$ had so far required a posterior capsulotomy. The leading cause of poor visual outcome was amblyopia. Two patients developed severe complications related to the intraocular lens.

Conclusions-Insertion of a lens implant at the time of cataract extraction appears to be well tolerated in the short term, and may offer significant advantages in an African setting.

(Br f Ophthalmol 2001;85:267-271)

Kenya

D Yorston

M Wood

London School of Hygiene and Tropical Medicine, London, UK A Foster

Correspondence to: Dr Allen Foster, London School of Hygiene and Tropical Medicine, Keppel Street, London WC1E 7HT, UK

a.foster@1shtm.ac.uk

Accepted for publication 2 November 2000

Cataract remains one of the most important avoidable causes of blindness in children. It has been estimated that there are 200000 children blind from cataract worldwide, and that 20 000-40 000 children are born with cataract every year. ${ }^{1}$ In developing countries the prevalence of blindness from cataract is thought to be about $1-4 / 10000$ children. This is about 10 times higher than the figure for industrialised countries. ${ }^{1}$ The lower prevalence of blindness in richer countries is probably the result of better management of cataract.
In east Africa cataract is now the leading cause of blindness in children. ${ }^{2}$ A recently published study from Uganda estimated that cataract was responsible for over $30 \%$ of all cases of blindness and visual impairment in children. Furthermore, the results of surgery for cataract in Uganda were poor. Of those children for whom follow up data were available, $56 \%$ had a corrected vision of less than $6 / 60 .^{2}$

One reason for the poor outcome of cataract surgery in Africa is the difficulty in obtaining full correction of refractive errors in young children. Contact lenses are expensive and impractical for most African families. Glasses get lost or broken, and are difficult to fit on infants and young children. Following encouraging reports of the use of hydrogel intraocular lenses in children, ${ }^{3}$ Kikuyu Eye Unit started to use lens implants for paediatric cataracts as a routine from 1993. This report is a retrospective study of the experience over the past 5 years.

\section{Patients and methods}

All patients under 11 years old, who had bilateral cataract and were treated with cataract surgery and insertion of an intraocular lens (IOL) between January 1993 and October 1997, were identified. Those with unilateral or traumatic cataracts were excluded. A total of 118 eyes in 71 patients were included in the study. (DY).

All surgery was carried out by one surgeon

Data were collected from patient files. Patients who had not been seen for 1 year or more, and who had a postal address, were requested to attend for a free follow up examination.

Visual acuities in preverbal children were measured with the Cardiff test cards. In children who could not cooperate, visual acuity was estimated using the child's ability to fixate on a small brightly coloured object.

All data were entered in a database (MS Access v 2.0)

\section{Results}

PREOPERATIVE

Over two thirds (69\%) of the patients were male. The average age of the patients at their first operation was 3.5 years. Twenty eight patients were under 2 years old at surgery, 20 were aged between 2 and 5, and 23 were over 5 years old at their first operation.

Three quarters of eyes had a preoperative visual acuity less than $3 / 60$ (Table 1). The preoperative visual acuity in eyes having surgery before 2 years of age was more likely to 
Table 1 Preoperative and postoperative visual acuity by length of follow up in 118 eyes having cataract surgery for non-traumatic bilateral cataract in childhood, Kenya

\begin{tabular}{lcclcccc}
\hline Acuity & Preop & $\begin{array}{c}<3 \\
\text { months }\end{array}$ & $\begin{array}{l}3-5 \\
\text { months }\end{array}$ & $\begin{array}{c}6-11 \\
\text { months }\end{array}$ & $\begin{array}{l}\text { 12-23 } \\
\text { months }\end{array}$ & $\begin{array}{l}\text { 24+ } \\
\text { months }\end{array}$ & Total \\
\hline $16 / 6-6 / 18$ & 0 & 2 & 4 & 5 & 11 & 22 & $44(37.3 \%)$ \\
$2<6 / 18-6 / 60$ & $20(16.9 \%)$ & 5 & 5 & 8 & 17 & 11 & $46(39.0 \%)$ \\
$3<6 / 60-3 / 60$ & $10(8.5 \%)$ & 0 & 0 & 0 & 0 & 2 & $2(1.7 \%)$ \\
$4<3 / 60$ & $88(74.6 \%)$ & 0 & 0 & 1 & 0 & 2 & $3(2.5 \%)$ \\
Not known & $0(0 \%)$ & 20 & 0 & 1 & 1 & 1 & $23(19.5 \%)$ \\
Total & $118(100 \%)$ & 27 & 9 & 15 & 29 & 38 & $118(100 \%)$ \\
\hline
\end{tabular}

be less than $3 / 60$ than older children $(\mathrm{p}<0.001)$ (Table 2). Of 118 eyes, 65 (55.1\%) had total cataracts, $46(39.0 \%)$ had lamellar or zonular cataracts, and in seven eyes the morphology was not recorded. Of 49 eyes under 2 years at surgery, $46(93.9 \%)$ had total cataracts compared with $19(30.7 \%)$ in those aged 2 years or more $(\mathrm{p}<0.001)$.

Fifty four of $71(76.1 \%)$ children were blind (less than $3 / 60$ in their better eye) preoperatively and $30(42.3 \%)$ had manifest nystagmus at the time of first surgery. Nystagmus was present in $75.0 \%$ of patients under 2 years old, and in $20.9 \%$ of patients aged 2 or older $(\mathrm{p}<0.001)$.

Out of 71 patients no cause for the cataract could be identified in 62 . Nine patients had autosomal dominant cataract and one patient with multiple ocular abnormalities may have had congenital rubella. Only seven children had other abnormalities apart from their cataracts. Most of these had varying degrees of psychomotor retardation. The other 64 patients appeared to have no other significant health problems apart from their poor vision.

SURGERY

Fifty seven eyes (48.3\%) had an anterior capsulotomy, followed by lens aspiration, with preservation of the posterior capsule and attempted lens placement in the capsular bag. The majority of these $(86.0 \%)$ were carried out in children over the age of 3 years. In 61 eyes $(51.7 \%)$, following anterior capsulotomy, the lens matter was removed by an automated vitrectomy instrument, followed by primary posterior capsulectomy and anterior vitrectomy. In most of these patients the lens implant was placed in the sulcus. Most of these operations $(91.8 \%)$ were carried out in children under 4 years old.

In 39 eyes, the anterior capsule was opened with a capsulorhexis, 22 had a linear capsulotomy, and in 57 the anterior capsule was removed with a vitrector. At the end of surgery, the lens implant was thought to be in the capsular bag in 68 eyes, in the sulcus in 40, and either bag/sulcus, or uncertain in 10 eyes. One eye suffered intraoperative posterior capsule rupture with vitreous loss.

Biometry was not available until June 1995. After that time, biometry was used in all children who could cooperate with keratometry. In practice, few children under 3 years old had biometry performed. Out of 118 eyes, biometry was performed in only 20 either due to non-availability or because the child was too small. The remaining eyes had a IOL implanted following these guidelines: under 1 year 28 dioptres; $1-2$ years old 26 dioptres; and over 2 years 24 dioptres.

Thirty six eyes had single piece hydrogel (Iogel, Alcon) IOL inserted. Eighty eyes had single piece polymethyl methacrylate (Aurolab, India) IOL implanted. Two eyes had three piece PMMA lenses (Aurolab, India) inserted. A standard adult sized IOL was used in all cases.

\section{POSTOPERATIVE}

The most common early postoperative complication was a severe fibrinous uveitis. This occurred in 36 (30.5\%) eyes, and was equally common whether the IOL was in the capsular bag or the ciliary sulcus. Most cases of severe uveitis occurred 4-8 days after surgery. Two patients had a decentred IOL which was repositioned. One patient had a persistent raised intraocular pressure, which settled on medical treatment. There were no cases of endophthalmitis.

Details of follow up and visual outcome are given in Table 1. Latest visual acuity measurements were carried out as follows: observation of fixation behaviour, 17 eyes; Cardiff test, 22 eyes; picture matching (LH test), 17 eyes; Snellen chart, 31 eyes. In eight eyes the method used was not recorded. The mean follow up time was 73.8 weeks post surgery (SD 69.6, SE 6.41 ), and the median follow up was 65 weeks. Twelve patients were Somalis, referred from refugee camps by the United Nations High Commissioner for Refugees, and it was particularly difficult to arrange follow up for this group of children. A follow up of 3 months or

Table 2 Latest visual acuity by age of surgery, in 118 eyes having non-traumatic cataract in childhood

\begin{tabular}{|c|c|c|c|c|c|c|c|c|c|c|c|c|c|c|c|c|}
\hline & \multicolumn{4}{|c|}{ Age 0-1 } & \multicolumn{4}{|c|}{ Age 2-5+ } & \multicolumn{4}{|c|}{ Age 6-10 } & \multicolumn{4}{|c|}{ Total } \\
\hline & \multicolumn{2}{|c|}{ Preop } & \multicolumn{2}{|c|}{ Postop } & \multicolumn{2}{|c|}{ Preop } & \multicolumn{2}{|c|}{ Postop } & \multicolumn{2}{|c|}{ Preop } & \multicolumn{2}{|c|}{ Postop } & \multicolumn{2}{|c|}{ Preop } & \multicolumn{2}{|c|}{ Postop } \\
\hline & No & $\%$ & No & $\%$ & No & $\%$ & No & $\%$ & No & $\%$ & No & $\%$ & No & $\%$ & No & $\%$ \\
\hline $6 / 4-6 / 18$ & 0 & 0 & 10 & 20.4 & 0 & 0 & 17 & 53.1 & 0 & 0 & 17 & 46.0 & 0 & 0 & 44 & 37.3 \\
\hline$<6 / 18-6 / 60$ & 1 & 2.0 & 27 & 55.1 & 9 & 28.1 & 8 & 25.0 & 10 & 27.0 & 11 & 29.7 & 20 & 16.9 & 46 & 39.0 \\
\hline$<6 / 60-3 / 60$ & 0 & 0 & 2 & 4.1 & 9 & 28.1 & 0 & 0 & 1 & 2.7 & 0 & 0 & 10 & 8.5 & 2 & 1.7 \\
\hline$<3 / 60$ & 48 & 98.0 & 2 & 4.1 & 14 & 43.8 & 1 & 3.1 & 26 & 70.3 & 0 & 0 & 88 & 74.6 & 3 & 2.5 \\
\hline Not known & 0 & 0 & 8 & 16.3 & 0 & 0 & 6 & 18.8 & 0 & 0 & 9 & 24.3 & 0 & 0 & 23 & 19.5 \\
\hline Total & 49 & 100 & 49 & 100 & 32 & 100 & 32 & 100 & 37 & 100 & 37 & 100 & 118 & 100 & 118 & 100 \\
\hline
\end{tabular}


more was available in 91 eyes $(77.1 \%)$ of 55 patients $(77.5 \%)$. The 55 patients with a minimum follow up of 3 months were similar in age, sex, and preoperative visual acuities to the 16 patients with less than 3 months of follow up. In children over 2 years old, eyes with immature or zonular cataracts were more likely to be followed up for 3 months or longer $(p<0.001)$. There was no difference in the frequency of intraoperative or early postoperative complications between the 91 eyes with at least 3 months of follow up, and the 27 eyes with less than 3 months of follow up.

The latest corrected acuity for all 118 eyes is shown in Table 2 . Ten out of 49 eyes operated before the age of 2 achieved a corrected acuity of $6 / 18$ or better, compared with 34 out of 69 eyes operated at the age of 2 or older $(\mathrm{p}<0.001)$.

In eyes with a minimum follow up of 3 months, 30 out of 40 eyes with lamellar cataracts obtained a vision of $6 / 18$ or better, as opposed to eight out of 44 eyes with total cataracts $(\mathrm{p}<0.001)$. Out of 55 patients who were followed up for a minimum of 3 months, 40 $(72.7 \%)$ were blind (less than $3 / 60$ in the best eye) preoperatively and only one (1.8\%) remained blind at their last follow up, although an accurate visual acuity could not be obtained in another three patients.

Five eyes $(5.5 \%)$ were known to have a poor outcome (best corrected acuity of less than $6 / 60$ ) at 3 month follow up. Three eyes were amblyopic, one eye had glaucoma, and one eye had a shallow anterior chamber and corneal oedema.

Out of 47 patients with a minimum follow up of 6 months, 21 (44.7\%) had nystagmus preoperatively. Only five $(10.6 \%)$ of these patients still had nystagmus at their last follow up. The remaining 16 patients had all developed central, steady, and maintained fixation. This was confirmed by repeated examinations by a trained orthoptist.

Sixty two eyes $(52.5 \%)$ had an intraoperative capsulotomy of which one $(1.6 \%)$ required a secondary capsulotomy. Of the 56 eyes which did not have an intraoperative capsulotomy, 21 have so far had a capsulotomy. Seven were carried out before 1 year postoperatively, five between 1 and 2 years, and nine at more than 2 years. Of 17 eyes with at least 2 year follow up only two $(11.7 \%)$ retained an intact posterior capsule.

Other late complications included distortion of the pupil, which occurred in seven eyes, six of which had Iogel lens implants. A fibrous membrane developed in front of the lens implant, following severe fibrinous uveitis, in five eyes. In all cases the membrane was removed surgically, and did not recur. Two eyes developed dense posterior synechiae, with a small, fixed pupil. In two eyes, the Iogel lens appeared to flex forwards, rather than posteriorly, and they suffered a shallow anterior chamber, with corneal oedema. Glaucoma was diagnosed in two eyes. Pupil capture of one side of the IOL optic occurred in one eye.

Out of 118 eyes, 37 (31.4\%) had asymmetric amblyopia (defined as a difference in visual acuity between the two eyes of at least $0.2 \mathrm{log}$ MAR units, or clear preferential fixation in one eye), of which 21 were operated before the child was 2 years old $(\mathrm{p}<0.05)$. Amblyopia occurred in 21 out of 49 second eyes operated, as opposed to 16 out of 69 first eyes $(p<0.05)$. Of 33 amblyopic eyes with a follow up of at least 3 months, 28 had a vision of $6 / 60$ or better. Amblyopia was managed by occlusion, which was supervised by an orthoptist working in the low vision clinic at Kikuyu Eye Unit. In those children who had adequate follow up to assess the effectiveness of occlusion, the difference in acuity between the two eyes was less than $0.2 \log$ MAR units in 11 , and 0.2 units or greater in eight.

Out of 55 patients with a follow up of at least 3 months, esotropia occurred in eight (14.5\%), seven of whom had their first operation before they were 2 years old. Exotropia occurred in 16 patients $(29.1 \%)$, and the remaining 31 $(56.4 \%)$ patients appeared to have normal alignment. In the 39 patients with a follow up of at least 1 year, $17(43.6 \%)$ remained orthotropic, six were esotropic $(15.3 \%)$, and 16 (41\%) exotropic. By 2 years, 15 of $23(60.9 \%)$ patients attending follow up had a squint.

Refraction was carried out at 1-2 months postoperatively, and thereafter once or twice every year. The results are given in Table 3 for

Table 3 Postoperative refractive error in 95 eyes at last attendance

\begin{tabular}{|c|c|c|c|c|c|c|}
\hline \multirow[b]{2}{*}{ Absolute spherical error (dioptres) } & \multicolumn{2}{|c|}{ No biometry $(n=79)$} & \multicolumn{2}{|c|}{ With biometry $(n=16)$} & \multicolumn{2}{|c|}{ Total $(n=95)$} \\
\hline & No & $\%$ & No & $\%$ & No & $\%$ \\
\hline \multicolumn{7}{|l|}{ Age group: } \\
\hline $0-0.99$ & 12 & 15.2 & 6 & 37.5 & 18 & 19.0 \\
\hline $1-1.99$ & 15 & 19.0 & 3 & 18.7 & 18 & 19.0 \\
\hline $2-2.99$ & 12 & 15.2 & 4 & 25.0 & 16 & 16.8 \\
\hline $3-3.99$ & 5 & 6.3 & 1 & 6.3 & 6 & 6.3 \\
\hline $4+$ & 35 & 44.3 & 2 & 12.5 & 37 & 38.9 \\
\hline Total & 79 & 100 & 16 & 100 & 95 & 100 \\
\hline Cylindrical error & No & $\%$ & No & $\%$ & No & $\%$ \\
\hline \multicolumn{7}{|l|}{ Age group: } \\
\hline $0-0.99$ & 24 & 30.4 & 5 & 31.3 & 29 & 30.5 \\
\hline $1-1.99$ & 17 & 21.5 & 8 & 50.0 & 25 & 26.3 \\
\hline $2-2.99$ & 17 & 21.5 & 3 & 18.7 & 20 & 21.1 \\
\hline $3-3.99$ & 11 & 13.9 & 0 & 0 & 11 & 11.6 \\
\hline $4+$ & 10 & 12.7 & 0 & 0 & 10 & 10.5 \\
\hline Total & 79 & 100 & 16 & 100 & 95 & 100 \\
\hline
\end{tabular}




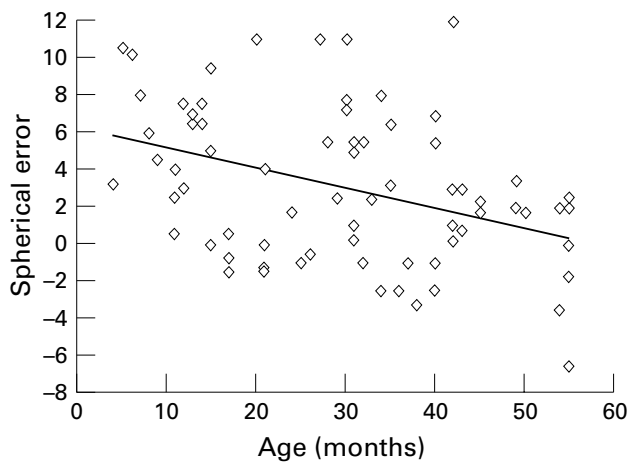

Figure 1 Spherical error in 20 eyes followed for 2 years.

95 eyes in which late refraction data are available. The average absolute spherical error was 3.81 dioptres (SD 3.57, SE 0.37). In eyes that had biometry, it averaged 2.15 dioptres, and in those that did not, it was 4.28 dioptres (Diff $\left.=2.13, \mathrm{SE}_{\mathrm{diff}}=1.01,95 \% \mathrm{CI}=0.13-4.13\right)$.

The spherical errors in 20 eyes operated before the age of 3 years, and with a minimum follow up of 2 years are plotted in Figure 1. Each eye was refracted between two and five times. The scatter plot shows a wide variation in refractive outcome, but a trend towards decreasing hypermetropia is visible.

Patients were provided with glasses as soon as possible, but it was sometimes difficult to obtain frames suitable for very young children. Furthermore, parents were often reluctant to pay for astigmatic and bifocal lenses, so some children received either no correction or incomplete correction.

\section{Discussion}

Blindness in children remains the second leading cause of blind person years worldwide. The prevalence of childhood blindness in Africa is approximately 10 times higher than in the industrialised nations. As half the population of sub-Saharan Africa is under 16 years, this represents a vast number of blind and visually impaired people. Special education for a blind child is too expensive for most African economies. Even when visually impaired children have attended blind school, they are rarely able to obtain employment in a highly competitive labour market.

Previously, the leading cause of blindness in east African children was corneal scarring, related to measles or vitamin A deficiency. ${ }^{45}$ However, recent advances in primary health care, particularly the success of the WHO/ UNICEF Extended Programme of Immunisation, have reduced the incidence of bilaterally blinding corneal scars. ${ }^{6}$ A study from Uganda ${ }^{2}$ has confirmed that cataract is now the leading cause of childhood blindness in east Africa.

In Uganda, $13.8 \%$ of cataracts were familial. In our own study, a similar proportion, $12.5 \%$, were inherited. We were unable to determine the aetiology in the other children. However, the absence of associated abnormalities makes major metabolic or chromosomal anomalies unlikely.

The preponderance of male patients is more likely the result of the greater value accorded to male children in traditional societies, rather than to an increased incidence of cataract in boys.

Most children operated before the age of 2 were blind with bilateral mature cataracts. In these children it was difficult to obtain accurate measurements of visual acuity, and our decision to operate was based on observation of the child's behaviour. If children could not recognise their mother, or developed nystagmus, we recommended surgery. If there was uncertainty, we preferred to wait until we were sure there was serious visual impairment.

No child was operated before the age of 3 months. This was partly because of late presentation, but also because of the risks of anaesthesia in very small infants, with no specialised paediatric anaesthetic service. This meant that many of our patients had established nystagmus. In children who were followed for 6 months or longer, the nystagmus resolved in $76.2 \%$ of those who had nystagmus preoperatively. This was an unexpected finding, and suggests that, in some children, the developing nervous system retains sufficient plasticity to overcome nystagmus, and develop steady fixation if vision is restored.

Although IOL fixation in the bag is superior to sulcus placement, it is difficult to achieve this in infants with a normal adult sized IOL. This may be because we use methylcellulose, whereas other authors have used more viscous solutions. Although there is a theoretically increased risk of uveitis with sulcus fixated lenses, we found that IOL placement did not affect the frequency of fibrinous uveitis. Fibrinous uveitis occurred in nearly one third of eyes. We cannot explain why uveitis should occur nearly a week after surgery in a previously quiet eye. It is possibly a reaction to the IOL material. In five eyes the uveitis left a fibrous membrane in front of the IOL, which could be removed easily and did not recur. Although the inflammation is a cause for concern, no patient in this series suffered permanent loss of vision because of uveitis.

We found an increased frequency of complications with Iogel lenses, particularly peaking of the pupil, which was severe in some patients. So far we have seen few complications with one piece PMMA lenses.

Posterior capsule opacification appears to be almost universal in children. A study in India showed that most children following lens aspiration will require capsulotomy. However, provided the child attended for follow up, and had a capsulotomy, the visual prognosis was good. ${ }^{7}$ Placing a biconvex IOL in the capsular bag might reduce the frequency of capsular opacification. However, in 17 eyes followed for at least 2 years, only two retained an intact posterior capsule. Our current practice is to perform primary posterior capsulectomy if follow up will be limited, or when it may be difficult to detect capsule opacification before it leads to amblyopia, particularly children under 5 years old.

Although biometry was available during the latter part of the study, it was of limited value, 
as most of our patients were unable to cooperate sufficiently to obtain accurate keratometry readings. There is such a large change in refractive error during the first few years of life that the value of biometry in infants and children under 2 years is questionable. Biometry was valuable in older children, and reduced the average absolute spherical error.

Poor follow up is a result of poverty. Although no fees are charged for children with cataracts, it is expensive for parents to bring children to the clinic. Secondly, most families in Kenya have several children. As education is costly, parents are reluctant to take scarce resources from their normal children, and use them for a child who may not become economically independent. Children with zonular or immature cataracts were more likely to attend follow up, as many of these children had quite good vision preoperatively, and their parents' decision to bring them for surgery indicated a higher level of parental commitment. We counsel parents preoperatively, so that they understand surgery is only the first stage of a long, and sometimes complex, rehabilitation programme.

The visual results are encouraging. Of eyes with a minimum follow up of 3 months, $91.2 \%$ had a latest corrected vision of $6 / 60$ or better. A corrected vision of $6 / 18$ or better was more likely in children with zonular cataracts, and in children operated after the age of 2 , as has been reported elsewhere. ${ }^{8}$ The worse results in younger children are most likely to be due to the greater frequency of mature cataracts in this age group, leading to more severe visual deprivation during the critical period of visual development. All three eyes with zonular cataracts that were operated before the age of 2 years achieved vision of $6 / 18$ or better. We advocate that surgery should be carried out as soon as possible in any child with severe visual loss or blindness from bilateral cataract.

Although results are slightly worse in children under 2 years old, with $85.9 \%$ of eyes having a vision of $6 / 60$ or better, this still compares favourably with the results from Uganda where $56 \%$ of eyes had a corrected vision of $6 / 60$ or better. ${ }^{2}$ The main reason for the poor outcome in Uganda was thought to be the failure to wear aphakic correction, which was also our experience in Kenya. While contact lenses represent a good method for correction of aphakia in infants and young children in developed countries, ${ }^{9}$ they are unsuitable for most households in Africa.

Two eyes $(1.7 \%)$ developed glaucoma. Poor follow up may have allowed other cases to go undetected. However, none of the children in this series had surgery before 3 months, and therefore they may be at less risk of glaucoma.

Two eyes became blind as a result of the IOL. These were small eyes, in which an Iogel lens was implanted. The lens flexed forwards rather than backwards, causing a shallow anterior chamber, corneal decompensation, and secondary glaucoma. No eyes have become blind following insertion of a single piece PMMA lens. However, the median follow up is only 65 weeks, and more complications may be seen with longer follow up.

Amblyopia was the leading cause of poor visual outcome. Most of the children operated before the age of 6 would have had some degree of amblyopia in both eyes. Asymmetric amblyopia was treated by occlusion. The major risk factors for amblyopia were surgery before the age of 2 years, and surgery on the second eye. In most cases second eye surgery is carried out 1 week after the operation on the first eye. As none of our cases were operated before the age of 3 months, it is unlikely that such a short interval could have caused the amblyopia. Amblyopia in the second eye might be the result of bias caused by operating first on the eye with better fixation.

The refractive outcome varied widely, regardless of the power of lens inserted. All patients who had surgery before the age of 3 showed a myopic shift in their refraction. If this continues, children who have a 28 dioptre IOL inserted at the age of 4 months may have significant myopia in later life. However, this is a small price to pay for an acceptable level of corrected visual acuity.

\section{Conclusion}

Cataract is now the leading cause of blindness in children in east Africa. The results of cataract surgery, with spectacle correction of aphakia, are poor. This study demonstrates that good results can be obtained in Africa using IOLs. In the short term, there appears to be little risk of blinding complications from the presence of an IOL. Only one child was still blind 3 months after surgery, compared with 40 preoperatively. We recommend that children needing surgery for cataract should be operated upon by an experienced surgeon who has access to safe paediatric anaesthesia. We advocate implantation of an IOL as the treatment of choice for most children with cataract in Africa.

1 Foster A, Gilbert C, Rahi J. Epidemiology of cataract in childhood: a global perspective. I Cataract Refract Surg 1997;23:601-4

2 Waddell $\mathrm{K}$. Childhood blindness and low vision in Uganda. Eye 1998;12:184-92.

3 Markham RH, Bloom PA, Chandna A, et al. Results of intra-ocular lens implantation in paediatric aphakia. Eye 1992;6:493-8

4 Faal H, Minassian D, Sowa S, et al. National survey of blindness and low vision in the Gambia: results. $\mathrm{Br} \mathcal{F} O \mathrm{ph}$ thalmol 1989;73:82-7.

5 Chirambo MC, Tielsch JM, West KP, et al. Blindness and visual impairment in southern Malawi. Bull World Health Organ 1986;64:567-72.

6 Foster A, Yorston D. Corneal ulceration in Tanzanian children: relationship between measles and vitamin A deficiency. Trans $R$ Soc Trop Med Hyg 1992;86:454-5.

7 Eckstein M, Vijayalakshmi P, Gilbert C, et al. Randomised clinical trial of lensectomy versus lens aspiration and primary capsulotomy for children with bilateral cataract in south India. Brf Ophthalmol 1999;83:524-9.

8 Taylor D. Congenital cataract: the history, the nature, and the practice. Eye 1998;12:9-36.

9 Lloyd I, Goss-Sampson M, Jeffrey B, et al. Neonatal cataract: aetiology, pathogenesis and management. Eye 1992;6:184-96. 